\title{
Expression of epithelial-Mesenchymal transition related markers in Plasmacytoid Urothelial carcinoma of the urinary bladder
}

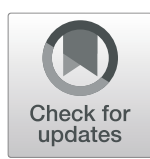

\author{
Shunichiro Nomura ${ }^{1 *}$ D, Yasutomo Suzuki ${ }^{1}$, Jun Akatsuka', Yuki Endo ', Akira Shimizu², Tsutomu Hamasaki', \\ Go Kimura ${ }^{1}$ and Yukihiro Kondo ${ }^{1}$
}

\begin{abstract}
Background: Plasmacytoid urothelial carcinoma (PUC) of the urinary bladder is a variant of urothelial carcinoma that carries a poor prognosis. The epithelial-mesenchymal transition (EMT) has been demonstrated to contribute to tumor progression. As the cause of the increased aggressiveness of PUC is unknown, we investigated PUC and EMT-related marker expression.

Methods: A total of 633 bladder carcinoma cases diagnosed from 2006 to 2015 at the Nippon Medical School Hospital were analyzed. Twelve patients were found to have plasmacytoid histology and diagnosed with PUC. Slides were evaluated for percentage of plasmacytoid variant, and stained for E-cadherin, N-cadherin, Vimentin, Fibronectin and Snail expression.
\end{abstract}

Results: The incidence of PUC was 1.9\% (12/633). The median patient age at diagnosis was 71 years (range, 60-80 years) and the male-female ratio was 11:1. All but three patients had stage T2b or higher. The median overall survival was 10 months. In 10/12 cases, Snail and N-cadherin were positive. Vimentin was positive in 9/12 cases. Fibronectin was positive in $8 / 12$ cases. While E-cadherin was negative in 10/12 cases. Nine cases showed $>10 \%$ plasmacytoid component. Eight of the nine patients (88.9\%) with $>10 \%$ plasmacytoid component died.

Conclusions: The results indicate that PUC may induce EMT and may be associated with high invasion.

Keywords: Bladder, Urinary bladder, Plasmacytoid, Urothelial carcinoma, E-cadherin, Snail, Epithelial-mesenchymal transition

\section{Background}

Plasmacytoid urothelial carcinoma (PUC) of the urinary bladder was first reported in 1991 as a similar histological type to plasma cell [1]. The World Health Organization (WHO) working group classified PUC as a variant of urothelial carcinoma (UC). These tumor cells have eosinophilic cytoplasm and eccentrically placed, enlarged hyperchromatic nuclei with small nucleoli [2].

\footnotetext{
* Correspondence: s-nomura@nms.ac.jp

'Department of Urology, Nippon Medical School, 1-1-5 Sendagi, Bunkyo-ku, Tokyo 113-8603, Japan

Full list of author information is available at the end of the article
}

The prognosis of PUC of the bladder is worse than that of conventional UC [3]. However, why PUC is more aggressive is unknown.

Later inquire about proposes that the epithelialmesenchymal transition (EMT) is a vital figure related to tumor progression and metastasis $[4,5]$. EMT could be a handle at first watched in embryonic improvement in which cells lose epithelial characteristics and pick up mesenchymal properties to extend motility and invasion [4]. Besides, a later ponder detailed that Snail could be a key controller of EMT [6]. Snail is a superfamily of zincfinger transcription factors that was, to begin with 
distinguished in Drosophila melanogaster [7]. Snail actuates EMT, in portion, by straighforwardly repressing epithelial markers such as E-cadherin and by upregulating mesenchymal markers such as N-cadherin, Vimentin, and Fibronectin. Immunohistochemistry has appeared downregulated or negative E-cadherin expression in the majority of PUC [3, 8]. Hence, PUC may actuate EMT. Therefore, EMT may be associated with PUC progression. The present study examined the expression status of EMT-related markers (E-cadherin, N-cadherin, Vimentin, Fibronectin and Snail) in PUC.

Whether survival is related to the proportion of plasmacytoid variant histology is unknown. Thus, we assessed the association between the proportion of plasmacytoid variant histology and survival in PUC patients. Furthermore, we also report clinical outcome information.

\section{Methods}

\section{Patients and samples}

The cohort under investigation comprised 12 patients who had bladder carcinoma with plasmacytoid histology at our institution between March 2006 and August 2015. All hematoxylin and eosin stained glass slides were retrieved and reviewed to confirm the diagnosis using the WHO definition of "plasmacytoid variants" [2]. Having been compiled for research purposes, this group represents patients for whom pretreatment archival paraffinembedded tissue blocks and data from complete clinical follow-up were available. Tumors were graded histologically in accordance with WHO classifications and were staged as per the TNM staging system of the Union for International Cancer Control (2009). The amount of PUC as a percentage was evaluated in the transurethral resection of bladder tumor (TURBT) and compared with that in the cystectomy when available.

\section{Immunohistochemistry}

Immunostaining was performed on at least one representative paraffin section using routine laboratory standard protocols. The antibodies used on paraffin-embedded tissues included EMA (Dako, Glostrup, Denmark), CK7 (Dako), CK20 (Dako), E-cadherin (Nichirei, Tokyo, Japan), N-cadherin (TaKaRa, Otsu, Japan), Vimentin (Dako), Fibronectin (Abcam, Cambridge, UK), Snail (Abcam) and CD138 (Dako). The stained tumor tissues were evaluated blindly with respect to clinical patient data. Staining was assessed using a semiquantitative scoring system $(0,1+$, $2+$, and $3+$ ). Immunohistochemical staining was evaluated as follows: 0 , no staining of tumor cells; $1+$, faint staining in less than $10 \%$ of tumor cells; $2+$, weak or moderate staining in more than $10 \%$ of tumor cells; and $3+$, strong staining in more than $10 \%$ of tumor cells. Staining intensity of 0 or $1+$ was considered negative, while $2+$ or $3+$ staining was considered positive. Negative controls were incubated without the primary antibody.

\section{Statistical analysis}

Associations between E-cadherin and IHC characteristics of PUC were analyzed using the Fisher's exact test. $P$-values $<0.05$ were considered significant. All statistical analyses were performed with SPSS Version 21.0 statistical software package (IBM Corp, Armonk, NY, USA).

\section{Results}

\section{Clinical data}

Among the 633 patients with bladder carcinoma that were initially identified, 12 were classified as PUC. Tumors diagnosed as PUC comprised 1.9\% (12/633) of all bladder carcinomas. The clinicopathologic features of the 12 patients are shown in Table 1 . The median patient age at diagnosis was 71 years (range, $60-80$ years) and there was only one female patient.

TNM stage was cT1N0 in three patients, cT2bN0 in three, cT3b-4aN0 in four, cT2bN1 in one, and cT3bN2 in one. The initial diagnosis of plasmacytoid carcinoma of the bladder was made on TURBT in 11 cases and cystoprostatectomy in one case. The pathologic $\mathrm{T}$ stage was pT1 in three patients, pT2b in seven, pT3b in one, and pT4a in one. None of the 12 cases had a prior history of bladder cancer.

The treatment outcomes of the 12 patients are shown in Table 2. One patient underwent radical cystectomy preceded by neoadjuvant chemotherapy. However, this surgical specimen was not pathologically free of cancer (pT2<). One patient underwent radical cystectomy alone, and two patients underwent palliative cystectomy. In all available specimens from patients who underwent

Table 1 Clinical and pathologic features of 12 cases of plasmacytoid urothelial carcinoma

\begin{tabular}{lllll}
\hline Case & Presenting & TNM & Pathologic & Plasmacytoid \\
No. & Symptoms & Clinical Stage & T stage & Component (\%) \\
\hline 1 & Hematuria & T3aN0M0 & $2 \mathrm{~b}$ & $>10$ \\
2 & Hematuria & T1N0M0 & 1 & $>10$ \\
3 & Hematuria & T2bN0M0 & $2 \mathrm{~b}$ & $>10$ \\
4 & Hematuria & T3bN0M0 & $3 \mathrm{~b}$ & $>10$ \\
5 & Frequent urination & T4aN0M0 & $4 \mathrm{a}$ & $>10$ \\
6 & incidental & T1N0M0 & 1 & $>10$ \\
7 & Residual urine & T3bN0M0 & $2 \mathrm{~b}$ & $<10$ \\
8 & Hematuria & T3bN2M0 & $2 \mathrm{~b}$ & $>10$ \\
9 & Hematuria & T2bN1M0 & $2 \mathrm{~b}$ & $>10$ \\
10 & Hematuria & T1N0M0 & 1 & $<10$ \\
11 & Hematuria & T2bN0M0 & $2 \mathrm{~b}$ & $>10$ \\
12 & Hematuria & T2bN0M0 & $2 \mathrm{~b}$ & $<10$ \\
\hline
\end{tabular}


Table 2 Treatment outcomes in 12 patients with plasmacytoid histology

\begin{tabular}{lllll}
\hline Case & & Post-treatment & Follow-Up \\
\cline { 3 - 4 } No. & Treatment & Progression site & Months & Status \\
\hline 1 & Radical cystectomy & Pelvic & 7 & Died of disease \\
3 & Chemotherapy & Retroperitoneal lymph nodes & 10 & Died of disease \\
4 & TURBT & Bladder & 9 & Died of disease \\
5 & Radical cystectomy & Lung & 5 & Died of disease \\
6 & Chemoradiation & Retroperitoneal lymph nodes & 9 & Died of disease \\
7 & Chemotherapy alone & No progression & 35 & Alive with disease \\
8 & TURBT & No progression & 36 & Alive with disease \\
9 & Radical cystectomy and Chemotherapy & No progression & 11 & Died of chemotherapy \\
10 & Chemotherapy & Retroperitoneal lymph nodes & 3 & Died of disease \\
11 & BCG & No progression & 30 & Alive with disease \\
12 & Radical cystectomy & Retroperitoneal lymph nodes & 3 & Died of disease \\
\hline
\end{tabular}

surgery, the negative margin and negative lymph node status rate at surgery were $75 \%$ (3 of 4 ) and $0 \%$ (0 of 2 ). Three patients received chemotherapy alone, and one of the three patients remained alive with disease and the other patient died of disease. All chemotherapy regimen was GC (gemcitabine and cisplatin). One patient received chemoradiotherapy alone and one patient received intravesical bacillus Calmette-Guerin immunotherapy alone. Two patients rejected any further treatment after TURBT. One patient did not receive any further treatment after TURBT due to a rapid worsening cancer.

At the time of analysis in August 2015, disease progression was objectively documented in 8 of the 12 patients. Disease recurrence was observed in the retroperitoneal lymph nodes in 5 of these 12 patients (42\%), while $1(8 \%)$ had recurrence in lung, and 1 patient each showed recurrence in bladder or pelvis.

Eleven patients had follow-up information available, while one was lost to follow-up. Eight of the patients died of their disease from 3 to 15 months (median 9 months), while three patients were alive from 29 to 36 months (median 36 months). One patient death was attributed to chemotherapy. With a median follow-up of 9.5 months, the overall median survival was 10 months. The 1-year survival rate was $33.3 \%$ for all patients.

Cases were stratified based on the percentage of plasmacytoid variant histology. Nine cases $(75 \%)$ showed > $10 \%$ plasmacytoid component, while three cases $(25 \%)$ showed $<10 \%$ plasmacytoid component. Eight of the nine $(88.9 \%)$ patients with $>10 \%$ plasmacytoid component died. The 1-year survival of patients was $11.1 \%$ (8/9 cases) for $>10 \%$ plasmacytoid patients. Conversely, the 1-year survival of patients was $100 \%$ (3/3 cases) for < $10 \%$ plasmacytoid patients.

\section{Immunohistochemistry}

The immunohistochemical findings in the 12 cases are summarized in Table 3. EMA was positive in 5/12 cases (41.7\%), CK7 was positive in 7/12 cases (58.3\%), CK20 was positive in only $2 / 12$ cases (16.7\%). E-cadherin was negative in 10/12 cases (83.3\%). N-cadherin and Snail were positive in $10 / 12$ cases $(83.3 \%)$. Vimentin was positive in $9 / 12$ cases (75\%). Fibronectin was positive in $8 / 12$ cases (66.7\%). There was no difference in these EMT-related markers expression between Ecadherin positive and negative PUC cells (Table 4). CD138 was negative in all cases. Snail was localized in the nucleus of PUC cells. E-cadherin and Ncadherin were localized in the cytoplasm of PUC cells. Typical UC was positive for E-cadherin. Representative cases of immunohistochemical staining are presented in Fig. 1.

\section{Discussion}

PUC is recognized as a rare and aggressive variant of UC, which often presents at a high stage and carries a poor prognosis [3]. Sahin et al. first described this tumor in 1991 [1]. Mai et al. reported an incidence of $2.7 \%$ of PUC in a series of muscle invasive UC [9]. In this study, 9 of 12 tumors were invasive. 9 of 12 PUC patients died during follow-up. The median age at diagnosis was 71 years, a finding similar to other recent reports. We recognized that the poor prognoses of these cases are due to the high invasion and the high clinical stage at presentation [3]. However, it is unclear how often PUC shows high invasion. 
Table 3 Summary of the immunostaining results

\begin{tabular}{|c|c|c|c|c|c|c|c|c|c|}
\hline Case No. & EMA & CK7 & CK20 & E-cadherin & $\mathrm{N}$-cadherin & Vimentin & Fibronectin & Snail & CD138 \\
\hline 1 & - & - & - & - & + & + & + & + & - \\
\hline 2 & + & - & - & - & + & + & + & + & - \\
\hline 3 & - & - & - & - & + & + & + & + & - \\
\hline 4 & - & + & - & - & + & + & + & + & - \\
\hline 5 & + & + & - & + & + & + & + & + & - \\
\hline 6 & - & + & + & + & + & - & - & + & - \\
\hline 7 & + & + & - & - & + & + & - & + & - \\
\hline 8 & - & - & - & - & - & + & - & + & - \\
\hline 9 & + & + & - & - & + & + & + & + & - \\
\hline 10 & - & - & - & - & - & - & - & - & - \\
\hline 11 & - & + & - & - & + & + & + & + & - \\
\hline 12 & + & + & + & - & + & - & + & - & - \\
\hline
\end{tabular}

EMT is an essential step amid epithelial tumor metastasis [10]. EMT causes changes in cell-cell and cellextracellular matrix interactions coming about in transmigration of cancer cells, in this way driving to metastasis $[11,12]$. E-cadherin may be a cell-cell intersection protein that is regularly downregulated or misplaced amid EMT, while expression of N-cadherin, Vimentin, Fibronectin, and Snail are obtained amid this process. In this study, E-cadherin expression was largely negative, while N-cadherin, Vimentin, Fibronectin and Snail were mostly positive. The present study reported a possibility of EMT in PUC.

E-cadherin is a key structure protein in maintaining both the stability of adhesion between epithelial cells and the stability of tissues [13]. Recent studies have demonstrated that loss of E-cadherin expression may correlate with high grade and advanced stage of UC $[14,15]$. Other studies reported that the majority of PUC cases show low E-cadherin expression [3, 8]. Keck et al. reported that most PUC with loss of membranous E-cadherin show a nuclear accumulation of E-cadherin. E-cadherin also serves as an independent prognostic factor for reduced overall survival of patients with muscle-invasive bladder cancer who were treated with radical cystectomy and adjuvant chemotherapy [16]. In this study, 10 of 12 cases $(83.3 \%)$ showed largely negative membranous Ecadherin.
Snail is considered a fundamental controller of EMT and, thus, of tumor movement. Bruyere et al. detailed that Snail expression predicts tumor recurrence in superficial bladder cancer [17]. Kosaka et al. reported that Snail expression might be a prognostic indicator of disease-free survival and disease-specific survival in upper urinary tract UC [18]. We moreover already detailed that Snail expression may anticipate poor results in bladder cancer patients treated with neoadjuvant chemotherapy [19]. In this study, Snail was mostly positive in $83.3 \%$. PUC also may predict the poor outcome by Snail. Further studies with a large cohort of PUC patients are needed to confirm this result.

Fibronectin is an essential component of extracellular matrix. It has been found highly expressed in a few kinds of cancer, indicating a potential role in progression [20, 21]. Malmstrom et al. reported the level of urine fibronectin in bladder cancer patients were significantly higher than that in patients with benign urothelial diseases and the health groups [22]. Furthermore, muscle invasive bladder cancer patients have a significantly higher level of urine fibronectin [23]. In this study, fibronectin was all negative in the patient had no progression. Although our study is the small number of cases, fibronectin may predict the recurrence of PUC patients.

Table $4 \mathrm{IHC}$ characteristics of PUC stratified according to the presence of E-cadherin

\begin{tabular}{|c|c|c|c|c|c|c|c|}
\hline & EMA neg. & CK7 neg. & CK20 neg. & N-cadherin pos. & Vimentin pos. & Fibronectin pos. & Snail pos. \\
\hline \multirow[t]{2}{*}{ E-cad. Neg. } & $6 / 10$ & $5 / 10$ & $9 / 10$ & $8 / 10$ & $8 / 10$ & $7 / 10$ & $8 / 10$ \\
\hline & $60 \%$ & $50 \%$ & $90 \%$ & $80 \%$ & $80 \%$ & $70 \%$ & $80 \%$ \\
\hline \multirow[t]{2}{*}{ E-cad. Pos. } & $1 / 2$ & $0 / 2$ & $1 / 2$ & $2 / 2$ & $1 / 2$ & $1 / 2$ & $2 / 2$ \\
\hline & $50 \%$ & $0 \%$ & $50 \%$ & $100 \%$ & $50 \%$ & $50 \%$ & $100 \%$ \\
\hline$P$ Value & 1 & 0.47 & 0.318 & 1 & 0.455 & 1 & 1 \\
\hline
\end{tabular}



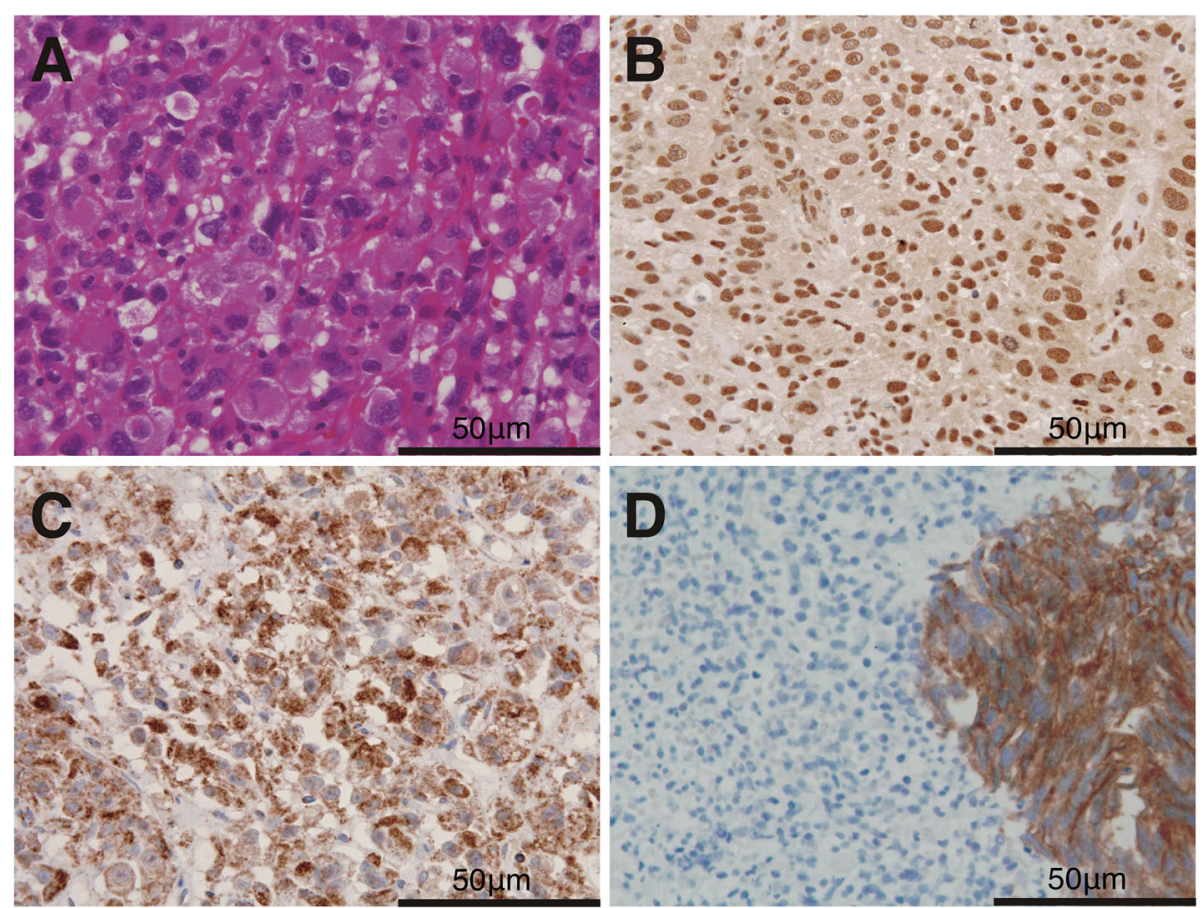

Fig. 1 a. Hematoxylin and eosin staining: the tumor cells have eosinophilic cytoplasm and eccentrically placed, enlarged hyperchromatic nuclei with small nucleoli. b. Snail-positive tumor cells. c. N-cadherin-positive tumor cells. d. E-cadherin-negative tumor cells of PUC with an E-cadherinpositive typical urothelial carcinoma

CD138 expression has been reported as a specific marker for PUC. However, Goto et al. reported the frequency of CD138 positivity in PUC was relatively low, compared with that observed in the conventional types and other variants [24]. In this study, CD138 was negative in all cases. Therefore, PUC should be correctly diagnosed using their characteristic cytomorphology.

Whether survival is related to the proportion of plasmacytoid variant histology has been unknown. Here we assessed the association between the proportion of plasmacytoid variant histology and survival in PUC patients. Analysis of the correlation between the amount of PUC and outcome revealed that the three patients with $<10 \%$ plasmacytoid component did not die from cancer, while eight of the nine patients $(88.9 \%)$ with $>10 \%$ plasmacytoid component died from disease. This result may demonstrate the importance in identifying the amount of PUC.

It remains unknown how PUC patients should be treated. Qiang et al. reported PUC was not associated with worse overall mortality compared with pure UC on multivariable analysis in a large cohort of patients treated with radical cystectomy [25]. Radical cystectomy may improve survival for patients with PUC. In this study, only four PUC patients underwent radical cystectomy and three patients underwent only TURBT.
Radical cystectomy should be considered as the first line choice for PUC.

\section{Conclusions}

Our study is the first to elucidate immunohistochemical evidence of EMT in PUC. PUC may induce EMT and may be associated with high invasion. More detailed studies are needed to address this question.

\section{Abbreviations}

PUC: Plasmacytoid urothelial carcinoma; WHO: World Health Organization; UC: Urothelial carcinoma; EMT: Epithelial-mesenchymal transition;

TURBT: Transurethral resection of bladder tumor

\section{Acknowledgments}

Kyoko Wakamatsu provided technical support.

\section{Authors' contributions}

SN evaluated immunohistochemical staining, performed the statistical analyses, and drafted the manuscript. YS assisted with the statistical analysis and helped draft the manuscript. JA and YE supervised the research and helped write the manuscript. AS evaluated the immunohistochemistry and helped draft the manuscript. TH revised the manuscript. GK did the histological review of the samples and reviewed the manuscript. YK participated in the study conception and design, data analysis, interpretation, drafting, and final approval of the manuscript. All authors have read and approved the final manuscript.

Funding

No funding was obtained for this study. 


\section{Availability of data and materials}

The datasets during and/or analyzed during the current study available from the corresponding author on reasonable request.

\section{Ethics approval and consent to participate}

The ethics committee of Nippon Medical School Hospital approved the study and patients have signed informed consent for the analysis and publication of their data.

\section{Consent for publication}

Not Applicable.

\section{Competing interests}

The authors declare that they have no competing interests.

\section{Author details}

'Department of Urology, Nippon Medical School, 1-1-5 Sendagi, Bunkyo-ku, Tokyo 113-8603, Japan. ${ }^{2}$ Analytic Human Pathology, Nippon Medical School, 1-1-5 Sendagi, Bunkyo-ku, Tokyo 113-8603, Japan.

Received: 22 January 2020 Accepted: 15 June 2020

Published online: 22 June 2020

\section{References}

1. Sahin AA, Myhre M, Ro JY, Sneige N, Dekmezian RH, Ayala AG. Plasmacytoid transitional cell carcinoma. Report of a case with initial presentation mimicking multiple myeloma. Acta Cytol. 1991;35(3):277-80.

2. Eble J, Sauter G, Epstein J. Pathology and genetics: tumours of the urinary system and male genital organs. . WHO Classification of Tumours. Lyon: IARC press; 2004

3. Keck B, Stoehr R, Wach S, Rogler A, Hofstaedter F, Lehmann J, Montironi R, Sibonye M, Fritsche HM, Lopez-Beltran A, et al. The plasmacytoid carcinoma of the bladder--rare variant of aggressive urothelial carcinoma. Int J Cancer. 2011;129(2):346-54.

4. Thiery JP. Epithelial-mesenchymal transitions in tumour progression. Nat Rev Cancer. 2002:2(6):442-54

5. Thiery JP, Chopin D. Epithelial cell plasticity in development and tumor progression. Cancer Metastasis Rev. 1999;18(1):31-42.

6. Guaita S, Puig I, Franci C, Garrido M, Dominguez D, Batlle E, Sancho E, Dedhar S, De Herreros AG, Baulida J. Snail induction of epithelial to mesenchymal transition in tumor cells is accompanied by MUC1 repression and ZEB1 expression. J Biol Chem. 2002;277(42):39209-16.

7. Boulay JL, Dennefeld C, Alberga A. The Drosophila developmental gene snail encodes a protein with nucleic acid binding fingers. Nature. 1987; 330(6146):395-8.

8. Lim MG, Adsay NV, Grignon DJ, Osunkoya AO. E-cadherin expression in plasmacytoid, signet ring cell and micropapillary variants of urothelial carcinoma: comparison with usual-type high-grade urothelial carcinoma. Mod Pathol. 2011;24(2):241-7.

9. Mai KT, Park PC, Yazdi HM, Saltel E, Erdogan S, Stinson WA, Cagiannos I, Morash C. Plasmacytoid urothelial carcinoma of the urinary bladder report of seven new cases. Eur Urol. 2006;50(5):1111-4.

10. Thiery JP, Acloque H, Huang RY, Nieto MA. Epithelial-mesenchymal transitions in development and disease. Cell. 2009:139(5):871-90.

11. Ghoul A, Serova M, Astorgues-Xerri L, Bieche I, Bousquet G, Varna M, Vidaud M, Phillips E, Weill S, Benhadji KA, et al. Epithelial-to-mesenchymal transition and resistance to ingenol 3-angelate, a novel protein kinase $C$ modulator, in colon cancer cells. Cancer Res. 2009:69(10):4260-9.

12. Nijkamp MM, Span PN, Hoogsteen IJ, van der Kogel AJ, Kaanders JH, Bussink J. Expression of E-cadherin and vimentin correlates with metastasis formation in head and neck squamous cell carcinoma patients. Radiother Oncol. 2011;99(3):344-8.

13. Baranwal S, Alahari SK. Molecular mechanisms controlling E-cadherin expression in breast cancer. Biochem Biophys Res Commun. 2009;384(1):611

14. Bringuier PP, Umbas R, Schaafsma HE, Karthaus HF, Debruyne FM, Schalken JA. Decreased E-cadherin immunoreactivity correlates with poor survival in patients with bladder tumors. Cancer Res. 1993;53(14):3241-5.

15. Syrigos KN, Krausz T, Waxman J, Pandha H, Rowlinson-Busza G, Verne J, Epenetos AA, Pignatelli M. E-cadherin expression in bladder cancer using formalin-fixed, paraffin-embedded tissues: correlation with histopathological grade, tumour stage and survival. Int J Cancer. 1995:64(6):367-70.

16. Keck B, Wach S, Kunath F, Bertz S, Taubert H, Lehmann J, Stockle M, Wullich B, Hartmann A. Nuclear E-cadherin expression is associated with the loss of membranous E-cadherin, plasmacytoid differentiation and reduced overall survival in urothelial carcinoma of the bladder. Ann Surg Oncol. 2013;20(7): 2440-5.

17. Bruyere F, Namdarian B, Corcoran NM, Pedersen J, Ockrim J, Voelzke BB, Mete U, Costello AJ, Hovens CM. Snail expression is an independent predictor of tumor recurrence in superficial bladder cancers. Urol Oncol. 2010:28(6):591-6.

18. Kosaka T, Kikuchi E, Mikami S, Miyajima A, Shirotake S, Ishida M, Okada Y, Oya M. Expression of snail in upper urinary tract urothelial carcinoma: prognostic significance and implications for tumor invasion. Clin Cancer Res. 2010;16(23):5814-23.

19. Nomura S, Suzuki Y, Takahashi R, Terasaki M, Kimata R, Hamasaki T, Kimura G, Shimizu A, Kondo Y. Snail expression and outcome in T1 high-grade and T2 bladder cancer: a retrospective immunohistochemical analysis. BMC Urol. 2013;13:73.

20. Ioachim E, Charchanti A, Briasoulis E, Karavasilis V, Tsanou H, Arvanitis DL, Agnantis NJ, Pavlidis N. Immunohistochemical expression of extracellular matrix components tenascin, fibronectin, collagen type IV and laminin in breast cancer: their prognostic value and role in tumour invasion and progression. Eur J Cancer. 2002;38(18):2362-70.

21. Rybak JN, Roesli C, Kaspar M, Villa A, Neri D. The extra-domain a of fibronectin is a vascular marker of solid tumors and metastases. Cancer Res. 2007;67(22):10948-57.

22. Malmstrom PU, Larsson A, Johansson S. Urinary fibronectin in diagnosis and follow-up of patients with urinary bladder cancer. Br J Urol. 1993;72(3):30710.

23. Menendez V, Fernandez-Suarez A, Galan JA, Perez M, Garcia-Lopez F. Diagnosis of bladder cancer by analysis of urinary fibronectin. Urology. 2005;65(2):284-9.

24. Goto K. CD138 expression is observed in the Urothelial epithelium and in various Urothelial carcinomas, and cannot be evidence for Plasmacytoid Urothelial carcinoma. Int J Surg Pathol. 2016;24(7):614-9.

25. Li Q, Assel M, Benfante NE, Pietzak EJ, Herr HW, Donat M, Cha EK, Donahue TF, Bochner BH, Dalbagni G. The impact of Plasmacytoid variant histology on the survival of patients with Urothelial carcinoma of bladder after radical cystectomy. Eur Urol Focus. 2019;5(1):104-8.

\section{Publisher's Note}

Springer Nature remains neutral with regard to jurisdictional claims in published maps and institutional affiliations.
Ready to submit your research? Choose BMC and benefit from:

- fast, convenient online submission

- thorough peer review by experienced researchers in your field

- rapid publication on acceptance

- support for research data, including large and complex data types

- gold Open Access which fosters wider collaboration and increased citations

- maximum visibility for your research: over $100 \mathrm{M}$ website views per year

At $\mathrm{BMC}$, research is always in progress.

Learn more biomedcentral.com/submissions 